\title{
Overview on optical measurements of shaft diameter detection
}

\author{
Yan Huang ${ }^{a}$, Chuande Zhou ${ }^{\text {b }}$ \\ School of Xi'an Shiyou University, Shanxi 710065, China \\ a990844100@qq.com, bzcd0013@163.com
}

Keywords: shaft diameter measurement, non-contact, optical method.

\begin{abstract}
Industrial development requires higher and higher shafts machining precision, which makes the quick, efficient and precise shafts measurement very important. Based on the Chinese and foreign literature review, this paper concluded the principle, method, accuracy, advantages, disadvantages and application range of common typical non-contact shafts measurement based on optical measurement. Associated detecting instruments were introduced and the further development trend of non-contact measurement was pointed out.
\end{abstract}

\section{Introduction}

As an important part of machine, shaft not only supports the rotor rotation and torque transmission, but also connects with rack through bearings ${ }^{[5]}$. It is one of most applied parts in various mechanical devices and plays an important role in machinery industry. Shaft diameter measurement, an important research area of measurement, is very important in measuring technique. Higher precision on shaft measurement were required with the development of measuring technique and increasing of production efficiency in enterprises. Shaft diameter measurement mainly includes contact measurement and non-contact measurement. In modern industry, contact measurement measures the shaft diameter through the contact of the measuring machine head and workpiece. Traditional contact measurement has a lot of shortcomings: 1) poor workpiece surface quality caused by the extrusion of the contact force; 2) low measurement accuracy; 3) unachievable dynamic measurement; 4) low measurement efficiency. However, non-contact measurement doesn't have these problems. Non-contact measurement method includes electronic non-contact measurement, optical non-contact measurement and others ${ }^{[4,8,15]}$. Among them, the electronic non-contact measurement is influenced by electromagnetic field significantly ${ }^{[6]}$. Ultrasonic non-contact measurement requires coupling medium on the probe and measuring surface, and the measuring result is determined by coupling conditions directly ${ }^{[3]}$. With the development of semiconductor laser device and photoelectric detector, optical non-contact measurement attracts more and more attentions. It has characters of high resolution, small influence from environmental electromagnetic field, long working distance, high accuracy of measurement, and applicability to non-metal surfaces and online measurement ${ }^{[9]}$. It is highly welcomed in modern industry and the current research hotspot.

\section{Optical non-contact measurement}

Most non-contact shaft diameter measurements are based on the principle of optical imaging projection. Instead of contact measurement, they measures image of shaft diameter, which won't be influenced by the measuring force ${ }^{[5]}$. Diffraction approach, laser scanning approach and image approach are commonly used in existing electro-optical diameter measuring instruments in China and foreign countries.

Diffraction approach. In the diffraction approach based on the principle of optical fluctuation, outgoing laser beam from the semiconductor laser device (LD) shines vertically onto the measuring thin cylinder on the XOY plane after passing through the diaphragm, and diffractive image field is accepted by CCD on the focal plane after the objective focusing. Diffraction signals accepted by CCD will be sent to the computer to calculate the shaft diameter ${ }^{[2]}$. 
Currently, common products using diffraction approach in China and foreign countries include Model1200 [13] (Laster mike Company, USA), LMDD-10 and LMDD-11 (Sichuan Huihuang Company). LMDD-10 is mainly used for accurate measurement of outer diameter (OD) of superfine wires (e.g. steel wire, copper wire and optical fiber). It has $0.005-0.5000 \mathrm{~mm}$ measurement range, $0.0001 \mathrm{~mm}$ measurement accuracy and 40 groups/s measuring speed.

To sum up, diffraction method is exclusive to measure small shafts. With characteristics of single-point measurement, intuitive and convenient measurement, low cost and easy online measurement, it is the most important accurate measuring method of superfine wires ${ }^{[7,11]}$. Due to these characteristics, diffraction method can only provide poor imaging quality and lower measurement accuracy when shaft diameter is larger than $0.5 \mathrm{~mm}$. Its measurement range is relatively narrow.
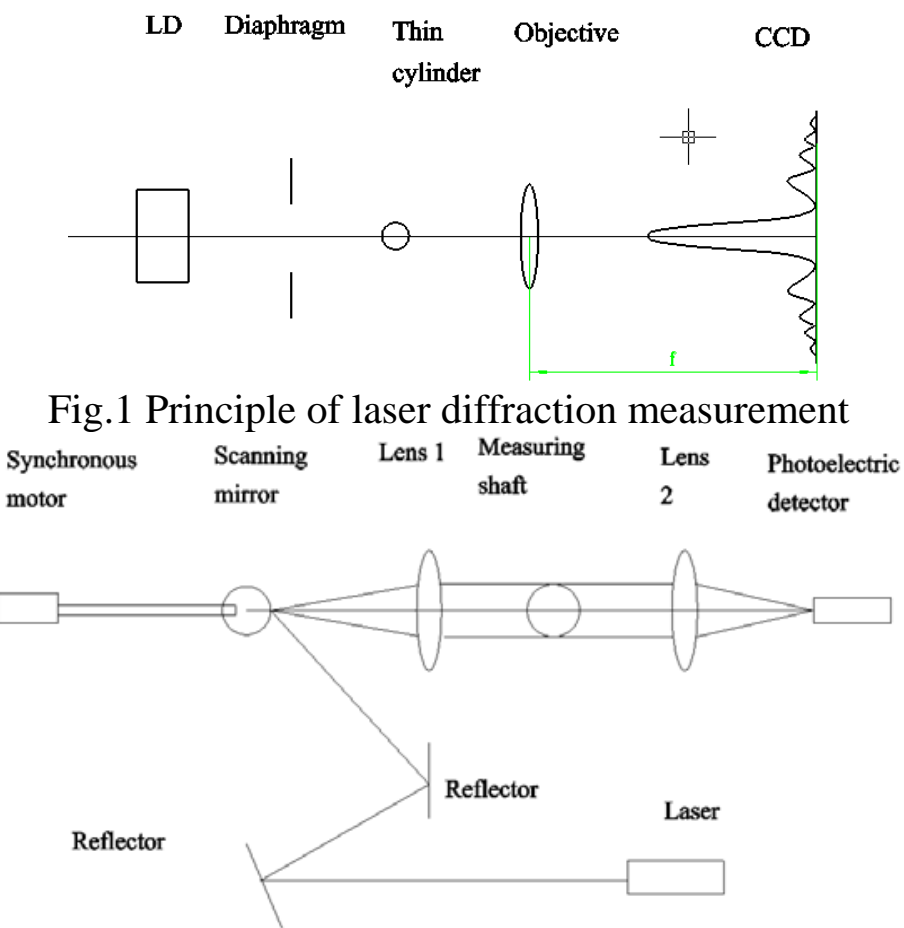

Fig.2 Measurement principle of laser scanning approach

Laser scanning approach. Laser scanning approach takes advantage of optical geometric principle (Fig.2). Beams emitted from the laser will pass through the reflector and then reflect onto the scanning mirror which makes constant-speed rotation driven by the synchronous motor. Consequently, the reflected beam scans optical lens 1 at a constant angular velocity, which turns the beam into parallel scanning beam and scans the measuring shaft. After passing through the optical lens 2, the beam is accepted by the photoelectric detector which will output a time-dependent optoelectronic signal. Later, the optoelectronic signal will be sent into computer to get the measured workpiece diameter after processed by following signal processing circuit ${ }^{[2]}$. Existing international products using laser scanning approach include American BetalaserMike's 510-195 (measurement range: 0.38 50mm; measuring repeatability: 0.0005mm), Tsi's SA5050 (measurement range: 1.0 48.0mm; measuring speed: *** times/s/shaft; measuring repeatability: 0.76 $\mu \mathrm{m}$; linearity: 0.01\%), and Japanese Kenyence's scanning LS-7030 series (measurement range: 0.3 30mm; uncertainty of measurement: $2 \mu \mathrm{m}$; measuring speed: 2400 times/s) ${ }^{[2]}$. Typical product using laser scanning approach in China is the JCJ-1 laser diameter measuring instrument made by Photoelectric Detection Department of Hebei Laser Research Institute. It has a measuring speed of 200 times $/ \mathrm{s}, 0.5 \mathrm{~mm}-60 \mathrm{~mm}$ measurement range and $\pm 0.01 \mathrm{~mm}$ measurement accuracy.

It can be seen from above data that laser scanning approach is characteristic of quick measurement and dynamic measurement. However, it still has some shortcomings due to the measuring equipment: 1) the expensive high-precision rotating mirror scanning system and micro motor increases the cost 
of the instrument; 2) it has nonlinear principle error which needs manual correction; 3) it will produce big errors when measuring shaft diameter smaller than $0.1 \mathrm{~mm}$.

Image approach. Image approach is also based on the optical geometric principle. It usually takes CCD as the photoelectric receiver. The principle of measurement is shown in Fig.3. The parallel light source will shine on the measuring shaft through the optical lens, while light blocked by the measuring shaft will form shadow on the CCD. The corresponding shadow position will output dark signal, while illuminated part forms carrier and outputs high level to transmit signal into computer. Diameter of the measuring shaft can be calculated from the pixels of shadow area ${ }^{[12,14,16]}$.

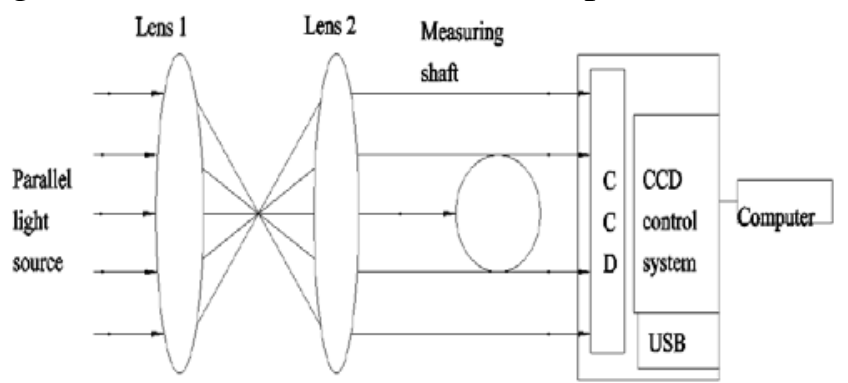

Fig.3 Measurement principle of image approach

Typical products using image approach include Japanese National's VS-6101A (measurement range was $1 \sim 25 \mathrm{~mm}$ and measurement uncertainty was $3 \mu \mathrm{m}$ and $5 \mu \mathrm{m}$ on shafts with diameter smaller and larger than 10mm respectively), Italian MARPOSS's OptoQuick Set, HOMMEL's AMV923, and TESA's TESA SCAN series. Typical products using image approach in China include ICMM series of Shanghai Jiaotong Unveristy and instrument for measuring lead width of integrated circuit board which is developed by Harbin Institute of Technology ${ }^{[2]}$.

Without complicated mechanical scanning unit in the laser scanning approach, the image approach has simple structure and low cost, thus applicable to large diameter measurement. However, when it is used for small shaft diameter measurement, it will cause serious diffraction, resulting in poor imaging quality and difficulties to determine shadow edges.

Comparisons on three approaches. The abovementioned three non-contact measurement approaches based on optical measurement are applicable to different situations for their different measurement modes, shown on table 1.

Table 1 Comparative analysis of three approaches

\begin{tabular}{ccccc}
\hline $\begin{array}{c}\text { Comparative } \\
\text { analysis }\end{array}$ & Range $(\mathrm{mm})$ & $\begin{array}{c}\text { Precision } \\
(\mathrm{mm})\end{array}$ & $\begin{array}{c}\text { Measuring } \\
\text { speed }\end{array}$ & Application scope \\
\hline $\begin{array}{c}\text { Diffraction } \\
\text { method }\end{array}$ & $0.005-0.5000$ & 0.0001 & 40group/s & Small diameter shaft $(\mathrm{d}<0.005 \mathrm{~mm})$ \\
\hline $\begin{array}{c}\text { Laser scanning } \\
\text { method }\end{array}$ & $0.5 \sim 60$ & 0.01 & 2400time/s & $\begin{array}{c}\text { Big shaft diameter, High speed } \\
\text { on-line measurement }(\mathrm{d}>0.5 \mathrm{~mm})\end{array}$ \\
\hline Image method & $1 \sim 25$ & 0.005 & $1.6 \mathrm{~s} /$ time & $\begin{array}{c}\text { Big shaft diameter, high precision } \\
\text { measurement }(\mathrm{d}>1 \mathrm{~mm})\end{array}$ \\
\hline
\end{tabular}

\section{Conclusions}

Industrial technological development puts forward higher requirements on product precision and measuring technique. Comparing with contact measurement, non-contact measurement is widely used for its workpiece protection, applicability to workpiece with complicated surface profile and quick measurement. Development trends of non-contact measurement methods based on the analysis of non-contact optical measurements in future can be concluded as:

High integration. Abovementioned measurement approaches are mainly focus on shaft diameter. They shall be developed to be multi-purpose device which can conduct online measurement of other parameters, such as surface roughness, concentricity and coaxiality. (2) High intelligence. These approaches not only have advantages and disadvantages, but also have different application environments and ranges. How to integrate them to develop a measurement instrument with 
intelligent judgment and optimal measurement selection is a key development direction. (3) Lower requirements on working environment. Most precise instruments are strict with working environment. Their measurement accuracy will be influenced under hostile environment. Therefore, it is important to develop a non-contact measurement immune to external environmental influence.

With the continuous development of optical technology, based on above research directions of non-contact measurement, future shaft measurement methods will develop toward higher efficiency, convenience and accuracy. Non-contact measurement based on the principle of optics will take the dominant role in measurement field.

\section{Acknowledgment}

Corresponding author: Chuande Zhou; School of Xi'an Shiyou University, Shanxi 710065, China; e-mail:zcd0013@163.com.

\section{References}

[1] Cheng Dalin. Design of Tran's missive Shaft Measurement System based on Laser Scanning [D]. Tianjin: Tianjin University, 2011.

[2] Cui Jianwen. Research on the Laser Diffraction-based Measurement of Thin Cylinder Diameter [D]. Harbin: Harbin Institute of Technology, 2007.

[3] Chen Kaiyun, Xie Xiaoqin. Manufacturing Technology \& Machine Tool, 2004, (8): 53) 56.

[4] Ferran Laguarta, Roger Artigas, Cristina Cadevall. Proc of SPIE, 2004, 5457: 74) 82

[5] Gao Fei. Research on Shaft Size Measurement based on Machine Vision Technology [D]. Changchun: Jilin University, 2007.

[6] Giovanna Sansoni, Alessandro Patrioli. Proc of SPIE, 2001, 4309: 232$) 239$.

[7]He X F, Liu W Y, Xu S D. Journal of Optoelectronics Laser,2005,16(7):821-824.

[8] Krissa Elizabeth Arn. Design of a non-cont act vibration measurement and analysis system $\mathrm{f}$ or electronic board testing[D] . United States Air Force Academy, 2004.

[9] Liu Feng. Research on the 3D Measurement of Free-form Surface based on the Principle of Structured Light Measurement [D]. Shenyang: Shenyang University of Technology, 2005.

[10] Luo Shengbin, Song Chunhua. Machine Tool \& Hydraulics, 2013.41(23):150-153.

[11]Moore E M, Shamhaugh RL, Papavassiliou D V.INJ Summer, 2004, 13(2): 44-47.

[12] Shang Chenghuo. Development of Data Acquisition System for CCD Precise Outer Diameter Measurement Instrument [D]. Wuhan: Wuhan University of Technology, 2010.

[13] Su Dongping. Research on Laser Diffraction Diameter Measurement Instrument based on EPGA [D]. Harbin: Harbin Institute of Technology, 2010.

[14] Sun Jian. Research on CCD Solid of Revolution Measurement Technique based on Linear Array [D]. Changchun: Changchun University of Technology, 2009.

[15] Sang Pil Youn, Young Kee Ryu. Proceedings of SPIE, 2001, 4190: 20$) 28$.

[16] Zhang Xiaoming. Research on the Automatic Measurement System of Small Shafts based on CCD [D]. Xi'an: Xi'an University of Technology, 2010. 